Proceedings of the Edinburgh Mathematical Society (2007) 50, 563-569 (C)

DOI:10.1017/S0013091505001161 Printed in the United Kingdom

\title{
WHEN THE SUM OF ALIQUOTS DIVIDES THE TOTIENT
}

\author{
WILLIAM D. BANKS ${ }^{1}$ AND FLORIAN LUCA ${ }^{2}$ \\ ${ }^{1}$ Department of Mathematics, University of Missouri, \\ Columbia, MO 65211,USA (bbanks@math.missouri.edu) \\ ${ }^{2}$ Instituto de Matemáticas, Universidad Nacional Autónoma de México, \\ C.P. 58089, Morelia, Michoacán, Mexico (fluca@matmor.unam.mx)
}

(Received 15 August 2005)

\begin{abstract}
Let $\varphi(\cdot)$ be the Euler function and let $\sigma(\cdot)$ be the sum-of-divisors function. In this note, we bound the number of positive integers $n \leqslant x$ with the property that $s(n)=\sigma(n)-n$ divides $\varphi(n)$.

Keywords: sum of divisors; Euler function; divisibility

2000 Mathematics subject classification: Primary 11A25

Secondary 11A41
\end{abstract}

\section{Introduction}

Let $\varphi(\cdot)$ be the Euler function:

$$
\varphi(n)=\#(\mathbb{Z} / n \mathbb{Z})^{*}=n \prod_{p \mid n}(1-1 / p), \quad n \geqslant 1 .
$$

Let $\sigma(\cdot)$ be the sum-of-divisors function,

$$
\sigma(n)=\sum_{d \mid n} d, \quad n \geqslant 1
$$

and let $s(n)=\sigma(n)-n$ be the sum of the aliquot divisors of $n \geqslant 1$. The function $s(n)$ and related arithmetic functions (such as $f(n)=n-\varphi(n)$ ) have been previously studied in the literature (see, for example, $[\mathbf{1}, \mathbf{2}, \mathbf{5}-\mathbf{8}]$ ).

In this note, we study the set of positive integers $n$ with the property that $s(n) \mid \varphi(n)$. Note that if $n$ is prime, then $s(n)=1$, and therefore $s(n) \mid \varphi(n)$; hence, we restrict our attention to composite integers $n$ with this property. Let

$$
\mathcal{B}=\{n \text { composite }: s(n) \mid \varphi(n)\} .
$$

Our main result is an unconditional upper bound for the counting function $\# \mathcal{B}(x)$ of the set $\mathcal{B}$, where $\mathcal{B}(x)=\mathcal{B} \cap[1, x]$. 
Theorem 1.1. The following estimate holds as $x \rightarrow \infty$ :

$$
\# \mathcal{B}(x) \leqslant x \exp \left(-\left(\frac{1}{3}(\log 8)^{1 / 3}+o(1)\right)(\log x)^{1 / 3}(\log \log x)^{2 / 3}\right) .
$$

We cannot show that $\mathcal{B}$ is an infinite set. However, if $m$ is a positive integer such that $p=5 m+1$ and $q=20 m+13$ are both primes, then for $n=p q$ we have $s(n)=25 m+15$ and $\varphi(n)=4 m(25 m+15)$, and therefore $n \in \mathcal{B}$. We recall that Dickson's prime $k$-tuplets conjecture [4] asserts that there are infinitely many examples of such pairs of primes $p$ and $q$. Thus, it is reasonable to expect that $\# \mathcal{B}=\infty$.

\subsection{Notation}

Throughout the paper, the letters $p$ and $q$ (with or without subscripts) are used to denote prime numbers, and the letter $n$ is used to denote a positive integer. As usual, we denote by: $P(n)$ the largest prime factor of $n ; \omega(n)$ the number of distinct prime factors of $n ; \Omega(n)$ the number of prime factors of $n$, counted with multiplicity; $\tau(n)$ the number of positive integer divisors of $n$; $\operatorname{ord}_{q}(n)$ the order at which the prime $q$ divides $n$ $\left(\operatorname{ord}_{q}(n)=\alpha\right.$ if and only if $\left.q^{\alpha} \| n\right)$.

For any set $\mathcal{A}$ of positive integers and a positive real number $x$, we put $\mathcal{A}(x)=\mathcal{A} \cap[1, x]$. We also put $\log x=\max \{\ln x, 1\}$, where $\ln (\cdot)$ is the natural logarithm, and we use $\log _{k}(\cdot)$ to denote the $k$ th iterate of $\log (\cdot)$. Finally, we use the Vinogradov symbols $\gg$ and $\ll$, as well as the Landau symbols $O$ and $o$, with their usual meanings.

\section{The proof of Theorem 1.1}

Let $x$ be a large real number, let $y=y(x)$ be a function of $x$ to be determined later, and put

$$
u=u(x)=\frac{\log x}{\log y} .
$$

In what follows, we assume that $y$ and $u$ tend to infinity with $x$.

Let us consider the following sets:

$$
\begin{aligned}
& \mathcal{B}_{1}(x)=\{n \in \mathcal{B}(x): P(n) \leqslant y\}, \\
& \mathcal{B}_{2}(x)=\left\{n \in \mathcal{B}(x) \backslash \mathcal{B}_{1}(x): P(n)^{2} \mid n\right\}, \\
& \mathcal{B}_{3}(x)=\left\{n \in \mathcal{B}(x) \backslash\left(\bigcup_{j=1}^{2} \mathcal{B}_{j}(x)\right): \omega(n)>u\right\}, \\
& \mathcal{B}_{4}(x)=\left\{n \in \mathcal{B}(x) \backslash\left(\bigcup_{j=1}^{3} \mathcal{B}_{j}(x)\right): \max _{q \mid n}\left\{\operatorname{ord}_{q}(n)\right\}>u(\log u) / \log 2\right\}, \\
& \mathcal{B}_{5}(x)=\left\{n \in \mathcal{B}(x) \backslash\left(\bigcup_{j=1}^{4} \mathcal{B}_{j}(x)\right): \omega(p-1)>u \text { for some prime } p \mid n\right\},
\end{aligned}
$$




$$
\begin{aligned}
& \mathcal{B}_{6}(x)=\left\{n \in \mathcal{B}(x) \backslash\left(\bigcup_{j=1}^{5} \mathcal{B}_{j}(x)\right): \max _{p|n, q| p-1}\left\{\operatorname{ord}_{q}(p-1)\right\}>u(\log u) / \log 2\right\} \\
& \mathcal{B}_{7}(x)=\mathcal{B}(x) \backslash\left(\bigcup_{j=1}^{6} \mathcal{B}_{j}(x)\right) .
\end{aligned}
$$

Since $\mathcal{B}(x)$ is the union of the sets $\mathcal{B}_{j}(x), j=1, \ldots, 7$, it suffices to find an appropriate bound on the cardinality of each set $\mathcal{B}_{j}(x)$.

We begin with the following well-known estimate of Canfield et al. $[\mathbf{3}]$ for the number of $y$-smooth numbers $n \leqslant x$ (see also $[\mathbf{9}]$ ):

$$
\Psi(x, y)=\#\{n \leqslant x: P(n) \leqslant y\}=x u^{-u+o(u)}, \quad u \rightarrow \infty
$$

which holds uniformly in the range

$$
(\log x)^{1+\varepsilon} \leqslant y \leqslant x^{1 / 3}
$$

for every fixed $\varepsilon>0$. From now on, we assume that (2.2) holds, and thus we have the bound

$$
\# \mathcal{B}_{1}(x) \leqslant x \exp (-(1+o(1)) u \log u)
$$

Next, let $n \in \mathcal{B}_{2}(x)$, and write $n=p^{2} m$, where $p=P(n)>y$ and $P(m) \leqslant p$. For each prime $p$ that arises in this way, the number of such integers $n \in \mathcal{B}_{2}(x)$ does not exceed $\left\lfloor x / p^{2}\right\rfloor ;$ therefore,

$$
\# \mathcal{B}_{2}(x) \leqslant \sum_{p>y} \frac{x}{p^{2}} \leqslant x \sum_{k>y} \frac{1}{k^{2}} \ll \frac{x}{y} .
$$

Put $K=\lfloor u\rfloor$. For every $n \in \mathcal{B}_{3}(x)$, there exist primes $p_{1}<\cdots<p_{K}$ such that $p_{1} \cdots p_{K} \mid n$. For every sequence of primes $p_{1}<\cdots<p_{K}$ arising in this way, the number of such integers $n \in \mathcal{B}_{3}(x)$ does not exceed $\left\lfloor x /\left(p_{1} \cdots p_{K}\right)\right\rfloor$; consequently,

$$
\# \mathcal{B}_{3}(x) \leqslant \sum_{\substack{p_{1} \cdots p_{K} \leqslant x, p_{1}<\cdots<p_{K}}} \frac{x}{p_{1} \cdots p_{K}} \leqslant \frac{x}{K !}\left(\sum_{p \leqslant x} \frac{1}{p}\right)^{K} .
$$

Using Stirling's formula together with Mertens's estimate

$$
\sum_{p \leqslant x} \frac{1}{p}=\log _{2} x+O(1)
$$

we see that the bound

$$
\# \mathcal{B}_{3}(x) \leqslant x\left(\frac{e \log _{2} x+O(1)}{\lfloor u\rfloor}\right)^{\lfloor u\rfloor}=x \exp (-(1+o(1)) u \log u)
$$

holds, provided that

$$
\log _{3} x=o(\log u) .
$$

From now on, we assume that (2.7) is satisfied. 
Let $L=\lfloor u(\log u) / \log 2\rfloor$. For every $n \in \mathcal{B}_{4}(x)$, there exists a prime $q$ such that $q^{L} \mid n$. Since the number of $n \in \mathcal{B}_{4}(x)$ divisible by $q^{L}$ does not exceed $\left\lfloor x / q^{L}\right\rfloor$, we have

$$
\# \mathcal{B}_{4}(x) \leqslant \sum_{q \leqslant x} \frac{x}{q^{L}} \leqslant x \sum_{k \geqslant 2} \frac{1}{k^{L}} .
$$

Since

$$
\sum_{k \geqslant 2} \frac{1}{k^{L}} \ll \int_{2}^{\infty} \frac{\mathrm{d} t}{t^{L}} \ll \frac{1}{2^{L}}=\exp (-(1+o(1)) u \log u),
$$

we derive the bound

$$
\# \mathcal{B}_{4}(x) \leqslant x \exp (-(1+o(1)) u \log u) .
$$

Let $K=\lfloor u\rfloor$ as before. For every $n \in \mathcal{B}_{5}(x)$, there exists a sequence of primes $p_{1}<\cdots<p_{K}$ and a prime $p \mid n$ such that $p_{1} \cdots p_{K} \mid p-1$. Write $p-1=p_{1} \cdots p_{K} \ell$, where $\ell \geqslant 1$; then the number of $n \in \mathcal{B}_{5}(x)$ divisible by $p$ is at most

$$
\left\lfloor\frac{x}{p}\right\rfloor \leqslant \frac{x}{p}<\frac{x}{p-1}=\frac{x}{p_{1} \cdots p_{K} \ell} .
$$

Summing over the possible choices of the primes $p_{1}<\cdots<p_{K}$ and the integer $\ell$, and applying Stirling's formula together with (2.5), we obtain

$$
\begin{aligned}
\# \mathcal{B}_{5}(x) & \leqslant \sum_{\substack{p_{1} \cdots p_{K} \ell \leqslant x, p_{1}<\cdots<p_{K}}} \frac{x}{p_{1} \cdots p_{K} \ell} \\
& \leqslant x\left(\sum_{\substack{p_{1} \cdots p_{K} \leqslant x, p_{1}<\cdots<p_{K}}} \frac{1}{p_{1} \cdots p_{K}}\right)\left(\sum_{\ell \leqslant x} \frac{1}{\ell}\right) \\
& \ll \frac{x \log x}{K !}\left(\sum_{p \leqslant x} \frac{1}{p}\right)^{K} \\
& \leqslant x \log x\left(\frac{e \log _{2} x+O(1)}{\lfloor u\rfloor}\right)^{\lfloor u\rfloor} \\
& =x \exp (-(1+o(1)) u \log u),
\end{aligned}
$$

where we have used (2.7) in the last step.

Let $L=\lfloor u(\log u) / \log 2\rfloor$ as before. For every $n \in \mathcal{B}_{6}(x)$, there exists a prime $p \mid n$ and a prime $q$ such that $q^{L} \mid p-1$. Write $p-1=q^{L} \ell$, where $\ell \geqslant 1$; then the number of $n \in \mathcal{B}_{6}(x)$ divisible by $p$ is at most

$$
\left\lfloor\frac{x}{p}\right\rfloor \leqslant \frac{x}{p}<\frac{x}{p-1}=\frac{x}{q^{L} \ell} .
$$

Summing over the possible choices of the prime $q$ and the integer $\ell$, we derive that

$$
\# \mathcal{B}_{6}(x) \leqslant \sum_{q^{L} \ell \leqslant x} \frac{x}{q^{L} \ell} \leqslant x\left(\sum_{q^{L} \leqslant x} \frac{1}{q^{L}}\right)\left(\sum_{\ell \leqslant x} \frac{1}{\ell}\right) \ll x \log x \sum_{k \geqslant 2} \frac{1}{k^{L}} .
$$


Using (2.8) together with (2.7), we derive the bound

$$
\# \mathcal{B}_{6}(x) \leqslant x \exp (-(1+o(1)) u \log u) .
$$

Finally, we come to the set $\mathcal{B}_{7}(x)$. Every integer $n \in \mathcal{B}_{7}(x)$ can be uniquely expressed in the form $n=p m$, where $p=P(n), y<p \leqslant x / m$, and $P(m)<y$. Thus,

$$
\# \mathcal{B}_{7}(x)=\sum_{m<x / y} \# \mathcal{P}_{m}
$$

where

$$
\mathcal{P}_{m}=\left\{p: p m \in \mathcal{B}_{7}(x) \text { and } p>P(m)\right\}, \quad m<x / y .
$$

Let $m<x / y$ be fixed in what follows, and suppose that $p \in \mathcal{P}_{m}$. We have

$$
s(p m)=\sigma(p m)-p m=p(\sigma(m)-m)+\sigma(m) .
$$

Since $p m$ is not prime (as it is an element of $\mathcal{B}$ ), $m \neq 1$, and hence $\sigma(m)-m>0$; therefore, the number $s(p m)$ determines $p$ uniquely. As $s(p m)$ is a divisor of $\varphi(p m)=$ $(p-1) \varphi(m)$, we can write $s(p m)=d_{1} d_{2}$ for some divisors $d_{1} \mid p-1$ and $d_{2} \mid \varphi(m)$. Reducing the identity (2.13) modulo $d_{1}$, it follows that $2 \sigma(m)-m \equiv 0\left(\bmod d_{1}\right)$ and, consequently,

$$
s(p m) \mid(2 \sigma(m)-m) \varphi(m) .
$$

In particular, for each $p \in \mathcal{P}_{m}$, all of the prime factors of the number $s(p m)$ lie in the set

$$
\mathcal{Q}_{m}=\{q: q \mid(2 \sigma(m)-m) \varphi(m)\}
$$

Using standard estimates, we have

$$
\# \mathcal{Q}_{m}=\omega((2 \sigma(m)-m) \varphi(m)) \leqslant\lfloor\log x\rfloor
$$

if $x$ is sufficiently large (in fact, $\left.\# \mathcal{Q}_{m} \ll(\log x) / \log _{2} x\right)$. On the other hand, for each $p \in \mathcal{P}_{m}$,

$$
\begin{aligned}
\omega(s(p m)) \leqslant \omega((p-1) \varphi(m)) & \leqslant \omega(p-1)+\omega(m)+\sum_{q \mid m} \omega(q-1) \\
& \leqslant 2 K+\sum_{q \mid m} L \leqslant 2 K+K L,
\end{aligned}
$$

where $K=\lfloor u\rfloor$ and $L=\lfloor u(\log u) / \log 2\rfloor$ as before. Let $\gamma(k)=\prod_{q \mid k} q$ denote the squarefree kernel of the integer $k$. Then the preceding argument shows that, as $p$ varies over the set $\mathcal{P}_{m}$, the number $\gamma(s(p m))$ takes at most

$$
\left(\begin{array}{c}
\lfloor\log x\rfloor \\
2 K+K L
\end{array}\right) \leqslant\left(\frac{\log x}{2 K+K L}\right)^{2 K+K L}
$$


possible values. Since the order at which any prime $q$ divides $s(p m)$ is bounded by

$$
\begin{aligned}
\operatorname{ord}_{q}(s(p m)) & \leqslant \operatorname{ord}_{q}((p-1) \varphi(m)) \\
& \leqslant \operatorname{ord}_{q}(p-1)+\operatorname{ord}_{q}(m)+\sum_{q^{\prime} \mid m} \operatorname{ord}_{q}\left(q^{\prime}-1\right) \\
& \leqslant 2 L+\sum_{q^{\prime} \mid m} L \leqslant 2 L+K L,
\end{aligned}
$$

we see that the total number of possibilities for the number $s(p m)$, as $p$ varies over the set $\mathcal{P}_{m}$, does not exceed

$$
\left(\frac{\log x}{2 K+K L}\right)^{2 K+K L}(2 L+K L)^{2 K+K L} .
$$

Since the number $s(\mathrm{pm})$ determines $p$ uniquely, we therefore obtain the bound

$$
\begin{aligned}
\# \mathcal{P}_{m} & \leqslant\left(\frac{\log x}{2 K+K L}\right)^{2 K+K L}(2 L+K L)^{2 K+K L} \\
& =\exp \left((2 K+K L)\left(\log _{2} x-\log (2 K+K L)+\log (2 L+K L)\right)\right) \\
& =\exp \left((1+o(1)) \frac{u^{2} \log u}{\log 2} \log _{2} x\right)
\end{aligned}
$$

where we have substituted the predefined values of $K$ and $L$ and used the fact that

$$
\log (2 L+K L)-\log (2 K+K L)=\log \left(\frac{2 L+K L}{2 K+K L}\right)=\log (1+o(1))=o(1)
$$

as $x \rightarrow \infty$. Using the previous estimate in (2.12), we deduce that

$$
\# \mathcal{B}_{7}(x)=\frac{x}{y} \exp \left((1+o(1)) \frac{u^{2} \log u}{\log 2} \log _{2} x\right) .
$$

Combining the estimates (2.3), (2.4), (2.6), (2.9)-(2.11) and (2.14), we have

$$
\# \mathcal{B}(x) \leqslant x \exp (-(1+o(1)) u \log u)+\frac{x}{y} \exp \left((1+o(1)) \frac{u^{2} \log u}{\log 2} \log _{2} x\right) .
$$

To optimize this estimate, we balance the two expressions by choosing the value of $u$ for which

$$
\frac{u^{2} \log u}{\log 2} \log _{2} x-\log y=-u \log u \text {. }
$$

In view of $(2.1)$, this is equivalent to

$$
\frac{u^{3} \log u}{\log 2} \log _{2} x+u^{2} \log u=\log x
$$

For such $u$, we have

$$
u=(1+o(1))\left((\log 8) \frac{\log x}{\log _{2} x}\right)^{1 / 3}
$$


and

$$
y=\exp \left(\left((\log 8)^{-1 / 3}+o(1)\right)(\log x)^{2 / 3}\left(\log _{2} x\right)^{1 / 3}\right) ;
$$

thus, $y$ and $u$ tend to infinity with $x$, and the required conditions (2.2) and (2.7) are clearly satisfied. With the above choice of $u$, we have

$$
\# \mathcal{B}(x) \leqslant x \exp (-(1+o(1)) u \log u)
$$

and the theorem follows.

Acknowledgements. This work was done during a visit by F.L. to the University of Missouri, Columbia; the hospitality and support of this institution are gratefully acknowledged. F.L. was supported in part by SEP-CONACyT Grant no. 46755 and a Guggenheim Fellowship.

\section{References}

1. W. D. Banks and F. LuCA, Nonaliquots and Robbins numbers, Colloq. Math. 103 (2005), 27-32.

2. J. Browkin and A. Schinzel, On integers not of the form $n-\phi(n)$, Colloq. Math. 68 (1995), 55-58.

3. E. R. Canfield, P. ERdős and C. Pomerance, On a problem of Oppenheim concerning 'factorisatio numerorum', J. Number Theory 17 (1983), 1-28.

4. L. E. Dickson, A new extension of Dirichlet's theorem on prime numbers, Mess. Math. 33 (1904), 155-161.

5. P. ERDős, Über die Zahlen der Form $\sigma(n)-n$ und $n-\phi(n)$, Elem. Math. 28 (1973), 83-86.

6. A. Flammenkamp and F. Luca, Infinite families of noncototients, Colloq. Math. 86 (2000), 37-41.

7. F. LuCA, On numbers not of the form $n-\omega(n)$, Acta Math. Hungar. 106 (2005), 117-135.

8. F. LUCA AND P. G. WALSh, On the number of nonquadratic residues which are not primitive roots, Colloq. Math. 100 (2004), 91-93.

9. G. Tenenbaum, Introduction to analytic and probabilistic number theory (Cambridge University Press, 1995). 